\title{
Pathological variations of lung masses
}

\author{
Neha Kakria ${ }^{1, *}$, Yadvir Garg ${ }^{2}$ \\ ${ }^{\mathbf{1}}$ Assistant Professor, Dept. of Radiodiagnosis, ${ }^{\mathbf{2}}$ Associate Professor, Dept. of Pulmonary Medicine, ${ }^{\mathbf{1} \mathbf{2}}$ Armed Forces Medical \\ College, Pune, Maharashtra, India
}

*Corresponding Author:

Email: neha.kakria15@gmail.com

Received: $24^{\text {th }}$, February, 2018

Accepted: $9^{\text {th }}$ April, 2018

\begin{abstract}
Introduction: Lung is a common place for the spread of tumors from within or other parts of the body. Lung shadows with a diameter less than or larger than $3 \mathrm{~cm}$ are termed lung nodule or mass respectively.

Aims: To study the histopathological spectrum of lung masses and efficacy of computerized tomography guided FNAC/biopsy in diagnosis of lung masses.

Materials and Methods: Forty (40) cases picked up as lung masses on conventional screening \& CT scan were subjected to CT guided FNAC/Biopsy and their pathological variations were studied.

Results: A total of 40 cases were studied. The mean age of the patients was 60.57 years with $25(62.5 \%)$ males and 15 (37.5\%) females. $65 \%$ of lesions were in right lung. Malignant lesions were more common and accounted for $27(67.5 \%)$ cases and benign in $8(20 \%)$ cases. Amongst the malignant lesions, adenocarcinoma was seen in $16(59.25 \%)$, squamous cell carcinoma in $10(37.03 \%)$ and small cell carcinoma in $1(3.70 \%)$ case.

Conclusion: FNAC is a simple, relatively safe, rapid, reliable technique for the diagnosis of pulmonary mass lesions, particularly with the aid of computed tomography (CT) scan. FNAC not only distinguishes between benign and malignant lesions but also helps in tumor typing of lung cancer.
\end{abstract}

Keywords: Lung mass, Computed tomography, Needle biopsy.

\section{Introduction}

Lungs, the most vital organs are exposed to both external and internal environment of the human body. It is thus prone to react to any adverse effects of the aetiological factors which may be infective, inflammatory, parasitic, vascular, neoplastic and other causes.

The detection \& diagnosis of any lung lesion usually begins with a chest radiograph, either in a symptomatic patient or a patient undergoing chest radiograph for an unrelated reason. Lung cancer was not even recognized as a distinct disease until 1761 . Different aspects of lung cancer were described further in 1810. Malignant lung lesions made up only $1 \%$ of all cancers seen at autopsy in 1878 , but had risen to 10 $15 \%$ by the early $1900 .{ }^{1}$ Worldwide in 2012, lung cancer occurred in 1.8 million people and resulted in 1.6 million deaths representing $19.4 \%$ of all deaths from cancer. $^{2}$ The American cancer society`s estimate for lung cancer in the United states for 2018 are: about 234,030 new cases $(121,680$ in men \& 112,350 in women), and about 154,050 deaths $(83,550$ in men 70,550 in women). Overall, the chance that a man will develop lung cancer in his life time is about 1 in 15 ; \& for a woman the risk is about 1 in $17 .^{3}$

CT of the chest introduced in the late 1970's, today remains the most widely used imaging test to stage patients with lung cancer. Definitive diagnosis however relies on the histopatholgical diagnosis of the tissue obtained by a CT guided FNAC, core biopsy or any other mode of biopsy.
A study of wide variety of pathology of lung masses based on cytology/histopathological diagnosis was undertaken at a tertiary care hospital.

\section{Materials and Methods}

A cross-sectional study of 40 patients who had reported with a suspicious pulmonary lesion on a chest radiograph was done at a tertiary care centre over a period of two years. These patients subsequently underwent CT scan imaging and CT guided FNAC /Core needle biopsy to arrive at a final diagnosis.

FNAC/Core Needle Biopsy: Informed consent of the patients was taken for CT guided FNAC/biopsy of the lung mass. The puncture entry point was infiltrated with $2 \%$ lignocaine. FNAC was performed using $22 \mathrm{G} / 23$ $\mathrm{G}$ spinal needle on 10 patients, CORE needle biopsy using a $18 \mathrm{G}$ automated core-biopsy needle on 29 patients and on 1 patient both FNAC and CORE needle biopsy had to be done for want of adequate and representative tissue. Air-dried smears were stained with May-Grünwald-Giemsa stain whereas alcoholfixed smears were stained with Papanicolaou stains for rapid cytopathological evaluation of the lesions. Core biopsy tissue was sent in (10\%) formalin solution to pathological lab and sections were stained by haematoxylin and eosin (H\&E) stain.

A follow-up CT scan was done on every patient immediately after the procedure to rule out pneumothorax or any haemorrhage. 


\section{Observations and Results}

The study was undertaken with the aim of studying the pathological variations of lesions presenting as suspicious lung masses. The computed tomographic observations of the lung masses were made with respect to location, size, margin, any associated calcification, cavitation, pleural effusion, mediastinal lymphadenopathy, character of contrast enhancement and bony involvement.

\section{General Considerations}

Age \& Sex: The mean age of the patients was 60.57 years with the standard deviation of 12.2284. There were $25(62.5 \%)$ males and $15(37.5 \%)$ females.

Majority of the lesions 26/40 (65\%) were in the right lung and $14 / 40(35 \%)$ in the left lung. Their size ranged from 3 to $10 \mathrm{~cm}$ with largest group comprising of 17 (43\%) having lung masses of the size $5-7 \mathrm{~cm}$. Fig. 1.

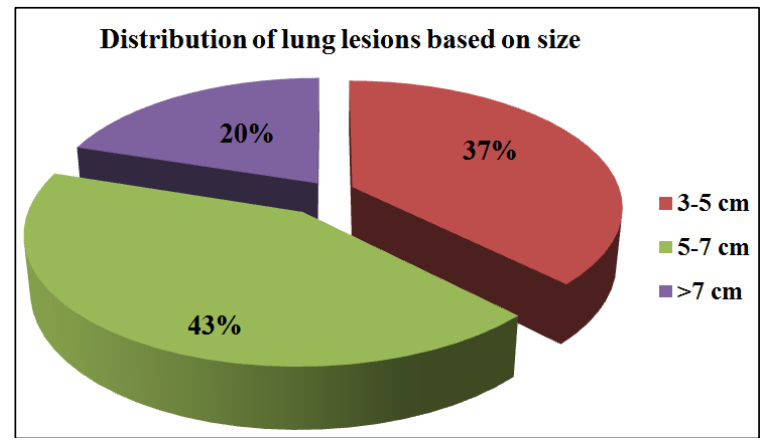

Based on radiological characteristics of the lesions alone, $35(87.5 \%)$ were provisionally diagnosed malignant and $5(12.5 \%)$ as benign.

The final cyto-pathological diagnosis was however malignant in $27(67.5 \%)$ cases and benign in $8(20 \%)$ cases. The remaining $5(12.5 \%)$ were inconclusive due to inadequate sampling.

Amongst the 27 (67.5\%), malignant lesions, adenocarcinoma was seen in $16(59.25 \%)$, of which mucin secreting adenocarcinoma is depicted in Fig. 4A, 4B and 4C. Squamous cell carcinoma was seen in 10 (37.03\%) patients. Poorly differentiated squamous cell carcinoma is as shown in Fig. 3A, 3B and 3C. Small cell carcinoma was seen in 1 (3.70\%) case. (Fig. 2).

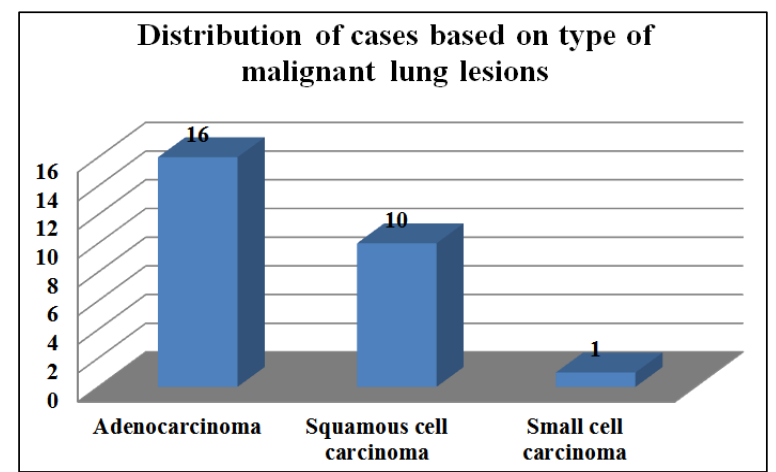

Fig. 2: Cyto-pathological diagnosis of malignant lesions

The distribution of benign lesions is shown in table 1 .

Table 1: Distribution of benign lesions

\begin{tabular}{|l|c|c|c|}
\hline S. No & Histopathological diagnosis & Number of cases & Percentage (\%) \\
\hline 1. & Granulomatous lesion lung & 02 & $25 \%$ \\
\hline 2. & Wegener's granulomatosis & 01 & $12.5 \%$ \\
\hline 3. & Mucormycosis & 01 & $12.5 \%$ \\
\hline 4. & Pneumonitis & 01 & $12.5 \%$ \\
\hline 5. & Pulmonary infarct & 01 & $12.5 \%$ \\
\hline 6. & Pulmonary hamartoma & 01 & $12.5 \%$ \\
\hline 7 & Fibrocollagenous tissue & 01 & $12.5 \%$ \\
\hline & Total & 08 & $100 \%$ \\
\hline
\end{tabular}

70 year old male, chronic smoker presented with anorexia and weight loss since 2 months

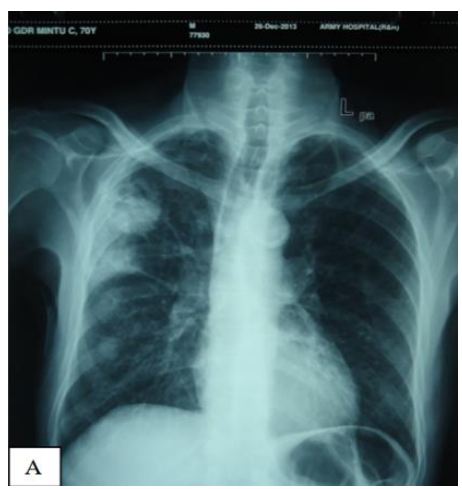

Fig. 3A: Chest XRay - Lobulated peripherally based mass lesion right middle zone. Associated incidentally old united fractures of right $\mathbf{4}^{\text {th }}$ and $\mathbf{9}^{\text {th }}$ ribs is seen posteriorly 

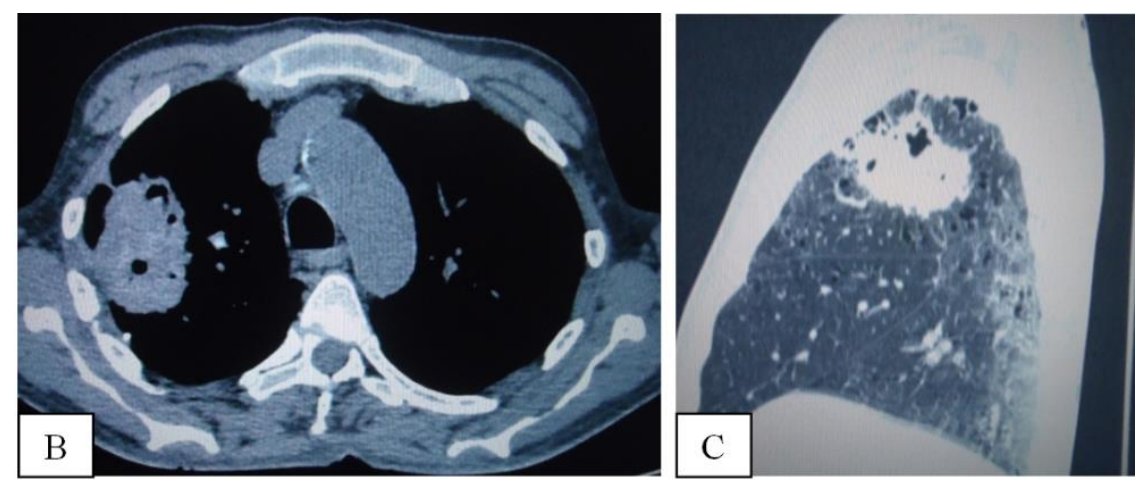

Fig 3B: CECT Chest mediastinal window axial section - Spiculated mass lesion apical segment of right upper lobe with irregular areas of breakdown within, suggestive of carcinoma right lung; C): CECT Chest lung window sagittal section - Spiculated mass lesion is seen in apical segment of right upper lobe

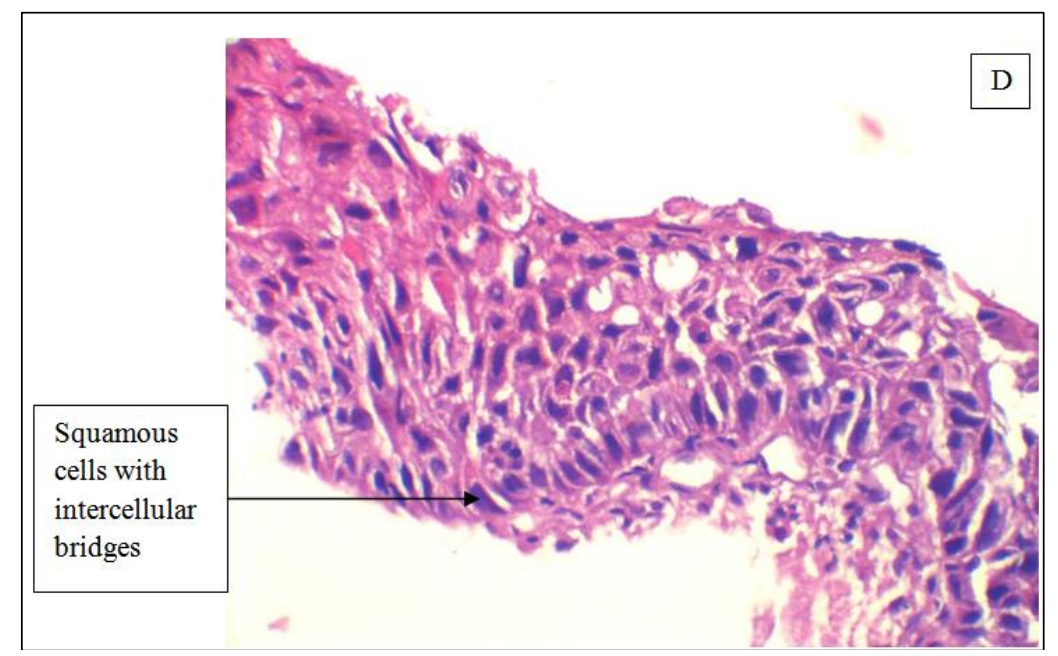

Fig. 3D: Biopsy/7434/13 - HPE shows malignant cells with high N:C ratio with abundant cytoplasm. Opinion:- Non small cell carcinoma- poorly differentiated squamous cell carcinoma.

45 year old female, chronic smoker, presented with right sided chest pain and hemoptysis.
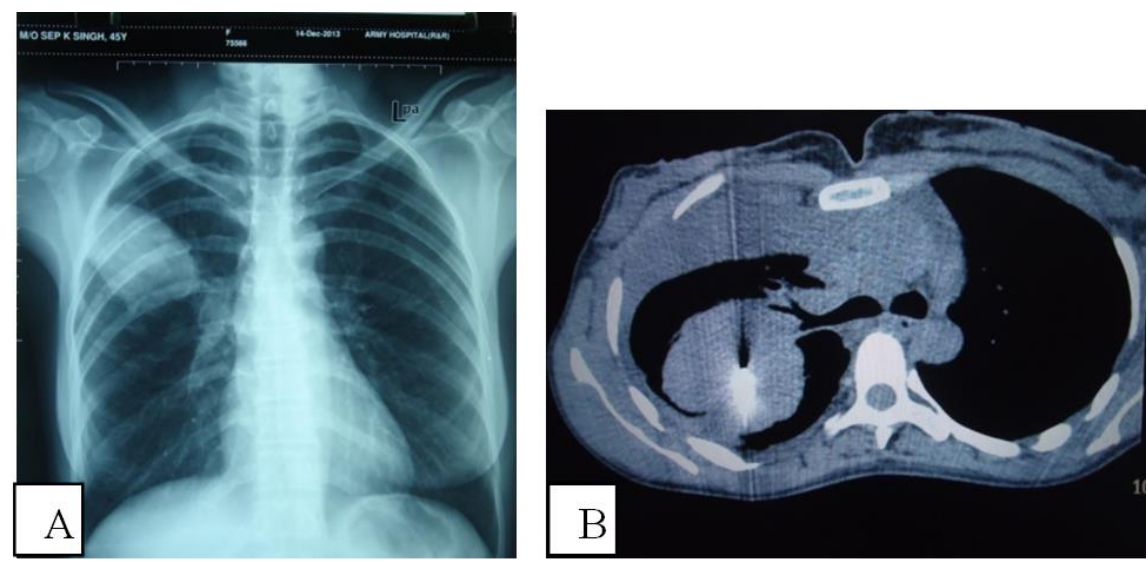

Fig 4 A\&B: Chest XRay - Mass lesion right upper zone. Fig. 14B: CT guided biopsy - Multilobulated mass lesion with biopsy needle tip seen in the lesion in posterior segment of right upper lobe, suggestive of carcinoma right lung. Right sided pleural effusion is also noted. 


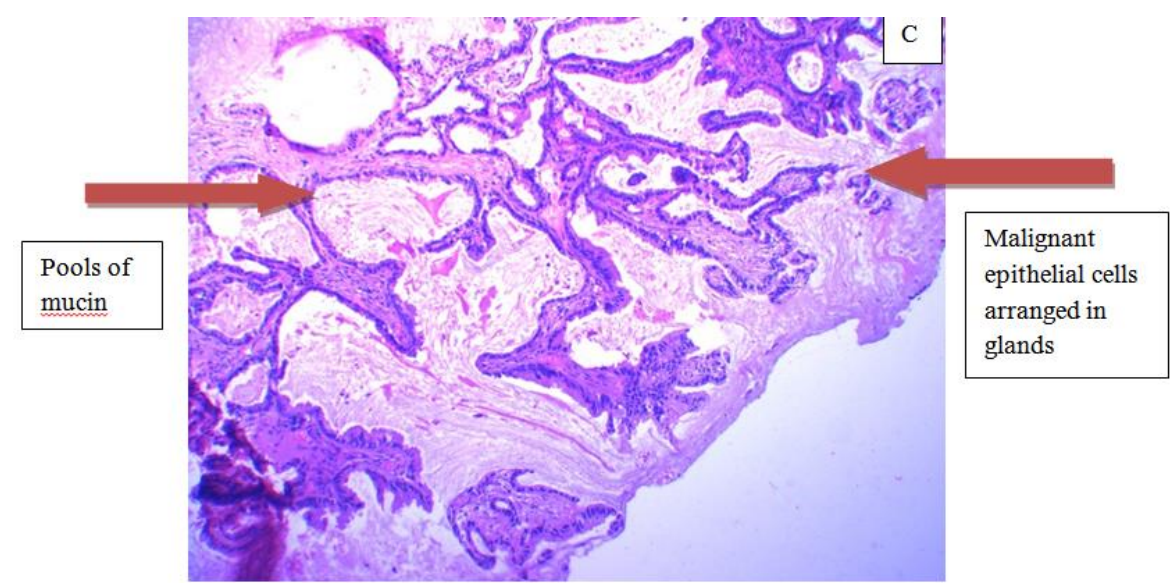

Fig. 4C: Biopsy/232/14 - HPE shows malignant epithelial cells arranged in glands with abundant pools of mucin amidst fibrocollagen

Opinion: Mucin secreting adenocarcinoma

In our study, cytology and HPE reports on FNAC/Core biopsy were considered as final confirmatory report. From a total of 35 cases in which adequate sampling was received; 30 were considered positive and 5 were considered negative for malignancy radiologically. False positive cases for malignancy based on radiology were 04 and false negative was 01 . Amongst the false positive cases, 01 was found to be pneumonitis as biopsy showed acute inflammatory cells with necrotic material, 02 cases were detected to be granulomatous lesions, (possibly of tubercular origin) and 01 was diagnosed as granulomatous polyangitis (Wegener's granulomatosis), as confirmed by HPE and immunohistochemistry (IHC).

The one (01) false negative case suspected to be fungal pneumonia radiologically turned out to be adenocarcinoma.

Chi square value (with Yates correction) was 7.35, and $p$ value 0.0066972 was statistically significant.

Complications: Pneumothorax occurred in 1 (2.5\%) case. It was minimal and got resolved without any treatment. Mild pulmonary haemorrhage occurred in 1 $(2.5 \%)$ case and $3(7.5 \%)$ cases complained of chest pain which got settled after one day.

\section{Discussion}

Diagnosis of lung mass lesions has always remained a challenging task. Any shadow more than 3 $\mathrm{cm}$ in size on chest X-ray or CT scan is suspected of malignancy unless proved otherwise. However, many benign disease processes such as infections, granulomatous disease, and cryptogenic organizing pneumonia may also present as a lung mass. Certain features can be depicted clearly with CT scan, which helps to suggest whether the lesion is benign or malignant. ${ }^{4}$ Transthoracic needle biopsy (FNAC/Core biopsy) using image guidance with $\mathrm{CT}$ has great diagnostic value for the definite characterization of lung lesions.

Numerous authors have expressed that the sensitivity and diagnostic accuracy is somehow slightly more in big lesions $(>3 \mathrm{~cm})$ and in lesions located peripherally. 5,6 Studies have also reported that cytological differentiation could be made properly in small cell and non-small cell lung cancers and there is good overlap in cytology and histopathological reports. ${ }^{15}$ Several factors can lead to non-specific biopsy results. The most common factors are tumor necrosis, inappropriate biopsy and insufficient sampling.

The most common complication of biopsy is pneumothorax. Kazerooni et al. showed that an increased lesion depth and small lesion size were strongly correlated with pneumothorax development. ${ }^{7}$ In our series, the parenchyma depth traversed for sampling was deeper in cases that developed pneumothorax.

In our study of 40 cases, on the basis of CT scan findings alone, a provisional radiological diagnosis about the nature of the lesion was made as malignant in $35(87.5 \%)$ and benign in $5(12.5 \%)$ cases. Histopathologically, out of 35, however $27(67.5 \%)$ proved to be malignant and $8(20 \%)$ cases were benign in nature. In 5 cases $(12.5 \%)$ results remained inconclusive due to insufficient sampling. Adenocarcinoma was the commonest comprising 16 $(59.25 \%)$ cases, squamous cell carcinoma $10(37.03 \%)$ cases, and small cell carcinoma only $1(3.70 \%)$ case. Benign lesions were $8(20 \%)$ with their diagnosis as granulomatous lesion (2 cases), and Wegener`s granulomatosis, mucomycosis, pneumonitis, pulmonary infarct, pulmonary hamartoma, fibrocollagenous tissue (1 case) each. We thus found a good overlap between the CT scan diagnosis and the final diagnosis as confirmed on cytology/histopathology. The most common age group was 51-70yrs with M:F ratio as 1.6:1. 
In a similar study of forty cases by Manas et al, ${ }^{8}$ the most common age group was 41-70yrs with male preponderance in the ratio of $\mathrm{M}: \mathrm{F}$ as 5:1.The predominant lesion found in the study was malignancy in 24 (60\%); followed by inflammatory lesions in 12 $(30 \%)$.One case each was reported as suspicious of malignancy $(2.5 \%)$ and granulomatous $(2.5 \%)$, the material was inadequate for interpretation in two cases $(5 \%)$. Out of 24 FNAC proven cases of malignancy, adenocarcinoma was found in $12(30 \%)$ followed by squamous cell carcinoma in $9(22.5 \%)$ cases and undifferentiated carcinoma in $3(7.5 \%)$ cases. The diagnostic accuracy was $95 \%$ considering cytological criteria as the standard.

Bahadir $\mathrm{T}$ et $\mathrm{al}^{9}$ in their study of 134 cases found the lesion at the mean age of 58.9 yrs. Maximum lesions were in the upper zone of right lung The final diagnosis of FNAC were malignant in 107 (80\%) cases, benign in $5(4 \%) \& 22(16 \%)$ were non-specific. The specificity for diagnosing malignancy was $100 \%$. adenocarcinoma was seen in $27(25 \%)$ cases, squamous cell carcinoma in $20(19 \%)$ cases.

Mukherjee, $\mathrm{S}$ et $\mathrm{al}^{10}$ in their study of 94 cases found malignancy in $90(\%)$ cases. The most common age group was 40-70 yrs with male preponderance (85\%). There was no false positive for malignancy. Hence there was $97.7 \%$ sensitivity and $100 \%$ specificity of CT guided FNAC as a diagnostic procedure. Over all accuracy for cytological typing was available in 88(95\%) cases. Non-small cell carcinoma predominated, similar to the experience of other workers.

Tan et $\mathrm{al}^{11}$ found the incidence of adenocarcinoma significantly higher than that of squamous cell carcinoma.

Jayashankar.E et $\mathrm{al}^{12}$ in their study of sixty cases found that majority of cases were in $5^{\text {th }}$ and $6^{\text {th }}$ decade and there was male preponderance. Provisional diagnosis based on radiologic findings was: 48 malignant and 12 cases benign. The cytological diagnosis however revealed 41 malignant and 19 benign. The diagnostic accuracy of CT guided FNAC was more than $90 \%$.The percentage distribution of different types of bronchogenic carcinomas was squamous cell carcinoma (51\%), adenocarcinoma (43\%), small cell carcinoma (3\%) and Poorly differentiated carcinoma( $3 \%)$.

Mini Gangopadhyay et $\mathrm{al}^{13}$ in their study of 127 cases found FNAC sensitivity as 96\% and 100\% specificity in diagnosing malignant lung tumours. Among the benign lesions, specific diagnosis was obtained in $48.1 \%$ cases. The males were $76(59.8 \%)$ and females $51(40.15 \%)$.The mean age was 58.71 yrs. 27 cases $(21.2 \%)$ were benign, 96 cases(75.6\%) malignant, 2 cases were suspected of malignancy and in another 2, the material was inadequate for cytopathological opinion. The most common carcinoma diagnosed by cytology was adenocarcinoma (54.2\%), followed by squamous cell carcinoma (25\%) and small cell carcinoma (8.3\%). In the benign lesions, 12 cases yielded tubercular granulomas, 12 cases as chronic nonspecific inflammation, 1 case of fungal infection (Aspergillus fumigatus) and 2 cases were of lung abscess.

Mondal SK, et al, ${ }^{14}$ in their prospective study of 130 cases analysed the radio-pathological correlation and found that that the peak incidence was in the fifth and sixth decade. The benign lesions were 10(8.07\%) and malignant lesions were $114(91.93 \%)$ as shown by cytology. The most common tumour was adenocarcinoma $(51.72 \%)$ followed by squamous cell carcinoma (6.89\%). Diagnostic accuracy of CT guided FNAC was $95 \%$. Post procedural complications like haemorrhage and chest pain were noted in only 3 cases.

Haresh chand et al ${ }^{15}$ in their series of retrospective study of 119 malignant cases found squamous cell carcinoma in 55(46.2\%), adenocarcinoma in 18 $(15.1 \%)$, Small cell carcinoma in $18(15.1 \%)$, Poorly differentiated carcinoma in $12(10.1 \%)$, Others $4(3.4 \%)$ and in $12(10.1 \%)$, the metastatic carcinoma. Male to female ratio was almost $1: 1$.The mean age group was 54.46 yrs. Upper zone of the right lung was the most common area involved in all the series. Various studies cite the incidence of malignant tumours on lung FNAC as $58-88 \% .^{2,5,7}$

\section{Conclusion}

The prevalence of lung mass is an extremely common finding that most radiologists will encounter on a frequent basis. Familiarity with the various manifestations of lung mass on chest radiography may help suggest the initial diagnosis. Once a suspicious abnormality is detected, CT guided FNAC/Core biopsy is the next step in the diagnostic work-up to arrive at a definitive diagnosis.

Literature reveals that $70 \%$ of the primary lung cancers are diagnosed as non-small cell carcinomas, whereas small cell carcinomas are rare and comprise around $20 \%$ of all primary lung tumours. ${ }^{7}$ In our study, the same pattern was observed. In the non-small cell group of carcinomas, adenocarcinoma remains the commonest tumor. Though squamous cell carcinoma used to be the commonest, studies indicate that adenocarcinomas may have overtaken it in incidence. ${ }^{15}$

FNAC which is not only a safe and rapid technique obviates the need for invasive techniques like mediastinoscopy, video-assisted thoracoscopy, and diagnostic thoracotomy in the diagnosis of pulmonary lesions. More so, initiation of specific therapy like chemotherapy or surgery is possible without unnecessary delay. When lung cancer is found at an early stage, the chance of cure is much higher than at later stages of the disease.

Conflicts of Interest: The author had none to declare. 


\section{References}

1. Witschi H. A short history of lung cancer. Toxicol Sci. 2001;64(1):4-6.

2. Cancer Report 2014.World Health Organisation.2014.11.Chapter 5.1.ISBN 92-832-04298.

3. Key Statistics for Lung Cancer-American Cancer Society, Jan 4, 2018.

4. Chaudhuri MR. Primary pulmonary cavitating carcinomas. Thorax 1973;28:354-366.

5. Stephen G.Spiro and Gerard A. Silvestri. "One hundred years of lung cancer". American Journal of Respiratory and Critical Care Medicine. 2005;172 (5):523-529.

6. Aoki T, Nakata H, Watanabe H, et al. Evolution of peripheral lung adenocarcinomas: $\mathrm{CT}$ findings correlated with histology and tumor doubling time. Am J Roentgenol 2000;174:763-8.

7. Kazerooni EA, Bhalla M, Shepard JA, McLoud TC. Adenosquamous carcinoma of the lung: radiologic appearance. Am J Roentgenol 1994;163:301-306.

8. Manas Madan, Hema Bannur. Evaluation of fine needle aspiration cytology in the diagnosis of lung lesions. Turkish journal of pathology 2010;26(1):16.

9. Bahadir Taha Uskul, Hatice Turker, Mertol Gokce, Aydin Kant, Sinan Arslan, Fatma Emre Turan. CTguided transthoracic fine needle aspiration of pulmonary lesions; Accuracy and complications in 134 cases. Tuberkuloz ve Toraks Dergisi 2009;57(2):177185.

10. Mukherjee Sumana, Bandopadhyay Gautam, Bhattacharaya Aparna et al. Computed tomographyguided fine needle aspiration cytology of solitary nodules suspected to be bronchogenic carcinoma.
Experience of a general hospital. Journal of Cytology 2010;27(1):8-11.

11. Tan KB, Thamboo TP, Wang SC, Nilson B, Rajwanshi A, Salto-Tellez M. Audit of transthoracic fine needle aspiration of the cytological subclassification of bronchogenic carcinoma and tuberculosis. Singapore Med J 2002;43:570-5.

12. JayShankar E, Pavani B, Chandra Eshwar, Reddy Ravinder, Srinivas M and Shah Ashwin. Computed tomography guided percutaneous thoracic: fine needle aspiration cytology in lung and mediastinum. Journal of cytology and histology 2010;1(3):107.

13. Gangopadhyay Mimi, Chakrabarti Indranil, Ghosh Nilanjana, Giri Amita. Computed tomography guided fine needle aspiration cytology of mass lesions of lung. Indian journal of medical and paediatric oncology 2011;32(4):192-196.

14. Mondal Santosh Kumar, Nag Dipanvita, Das Rama, Mandal Palash Kumar, Biswas Pranab Kr, Osta Manish. Computed tomogram guided fine-needle aspiration cytology of lung mass with histological correlation: A study in eastern India. South Asian Journal of Cancer 2013;2(1):14-18.

15. Haresh Chand, Khursheed Ahmed Abbasi, Saeed Ahmed Shaikh, Raja Rahul Chand. Pattern of Lung Tumors at Chandka Medical College \& Hospital Larkana: PJMHS 2011:5(1):15-19.

How to cite this article: Kakria N, Garg Y. Pathological variations of lung masses. Ind J Pathol Oncol, 2018;5(3):435-440. 